\title{
Acute 5-HT Reuptake Blockade Potentiates Human Amygdala Reactivity
}

\author{
Kristin L Bigos*,', Bruce G Pollock ${ }^{2,3}$, Howard J Aizenstein ${ }^{3}$, Patrick M Fisher ${ }^{4}$, Robert R Bies ${ }^{1,3}$ and \\ Ahmad R Hariri ${ }^{3}$ \\ 'Department of Pharmaceutical Sciences, University of Pittsburgh, PA, USA; ${ }^{2}$ Centre for Addiction and Mental Health, Rotman Research Institute, \\ University of Toronto, ON, Canada; ${ }^{3}$ Department of Psychiatry, University of Pittsburgh, PA, USA; ${ }^{4}$ Center for Neuroscience, University of \\ Pittsburgh, PA, USA
}

\begin{abstract}
Variability in serotonin (5-HT) function is associated with individual differences in normal mood and temperament, as well as psychiatric illnesses, all of which are influenced by amygdala function. This study evaluated the acute effects of 5-HT reuptake blockade on amygdala function using pharmacological functional MRI. Eight healthy men completed a double-blind balanced crossover study with the selective 5-HT reuptake inhibitor, citalopram $(20 \mathrm{mg}$ infused over $30 \mathrm{~min}$ ), and normal saline. Amygdala reactivity in response to novel facial expressions was assessed on three successive scans, once before drug/placebo infusion, once early in the infusion, and once at the end of infusion. Acute citalopram administration resulted in concentration-dependent increases in human amygdala reactivity to salient stimuli. The current pattern of 5-HT-mediated amygdala reactivity may represent an important pathway through which SSRIs achieve an antidepressant effect. Intriguingly, our data may also reveal a mechanism contributing to clinical observations of extreme agitation, restlessness, and suicidal ideation in some individuals during acute SSRI treatment. Developing a comprehensive model of how 5-HT modulates human amygdala reactivity supporting behavioral and physiological arousal will be instrumental for our understanding of basic neurobehavioral processes, their dysfunction in psychiatric illnesses, and their contribution to mechanism of treatment response. Neuropsychopharmacology (2008) 33, 3221-3225; doi: I 0. I038/npp.2008.52; published online 7 May 2008
\end{abstract}

Keywords: citalopram; SSRI; serotonin; amygdala; functional MRI; healthy subjects

\section{INTRODUCTION}

The amygdala is an evolutionarily conserved deep brain structure uniquely capable of mediating both simple reflexive and more complex adaptive behavioral and physiologic responses to environmental challenge. Moreover, variability in the functional dynamics of the amygdala has been associated with a broad range of individual differences in normal mood and temperament, as well as pathological emotional states such as depression. Accordingly, identifying the mechanisms regulating amygdala function and, in turn, mediating the emergence of such inter-individual variability is a major focus of neurobiological, psychological, pharmacological, and genetic research.

Converging evidence from these and other disciplines has highlighted the importance of serotonin $(5-\mathrm{HT})$ in the modulation of amygdala function and related behavioral processes. Available data from animal studies indicate that relative increases in amygdala 5-HT result in potentiation of

*Correspondence: Dr KL Bigos, Clinical Brain Disorders Branch, National Institute of Mental Health, 10 Center Drive, Room 3CIOI, Bethesda, MD 20892, USA, Tel: + I 301594 2429, Fax: + | 301480 0169, E-mail: bigosk@mail.nih.gov

Received 2 November 2007; revised 5 March 2008; accepted 6 March 2008 amygdala activation and associated behavioral phenomenon, such as fear conditioning (Burghardt et al, 2004, 2007; Forster et al, 2006). In humans, recent in vivo neuroimaging studies have revealed that decreased capacity for either 5-HT reuptake (Rhodes et al, 2007) or negative feedback autoregulation (Fisher et al, 2006) result in relatively increased amygdala reactivity. In addition, human functional genetic polymorphisms resulting in relatively increased 5-HT signaling are similarly associated with increased amygdala reactivity (Hariri et al, 2002; Heinz et al, 2005; Meyer-Lindenberg et al, 2006).

While such indirect evidence suggests similar potentiating effects of 5-HT in humans, the direct effects of increased 5-HT on human amygdala reactivity remain unexplored. In the current study, we used pharmacological functional MRI (fMRI) to examine the direct effects of acute 5-HT reuptake blockade on human amygdala reactivity. Consistent with the prior preclinical and clinical findings, we hypothesized that acute 5-HT reuptake blockade would result in heightened amygdala reactivity in comparison to a placebo condition.

\section{SUBJECTS AND METHODS}

All subjects signed a University of Pittsburgh Institutional Review Board-approved consent form prior to any research 
procedures. Eight right-handed, nonsmoking, Caucasian men (mean age 28 years, range 19-50 years) completed the study. These selection criteria were designed to minimize between-subject variation and possible age-related and ethnic differences in fMRI response (Aizenstein et al, 2004). Subjects were physically and psychiatrically healthy, as verified by the Structured Clinical Interview for DSM-IV disorders (First et al, 1995).

Subjects completed an archival fMRI challenge paradigm (Hariri et al, 2002) during a double-blind balanced crossover of intravenous (i.v.) citalopram, the most selective SSRI available, and normal saline. Subjects were randomized to placebo or citalopram treatment and then crossed over to the other treatment. Four subjects received citalopram on their first visit and four subjects received placebo first. Visits were separated by a minimum washout period of 2 weeks. Amygdala reactivity in response to novel neutral and affective facial expressions was assessed on three successive scans during both treatments. The challenge paradigm was performed once before drug/ placebo infusion (baseline), once early in the infusion, and once at the end of infusion when citalopram concentrations approach their maxima.

\section{Citalopram}

Parenteral citalopram (20 mg infused over $30 \mathrm{~min}$ ) was administered under an FDA investigational license. Citalopram was obtained from Lundbeck (Copenhagen, Denmark). Given the timing of the fMRI scans (pre-infusion, early infusion, and end of infusion), an optimal sampling strategy was determined using the D-optimal sampling algorithm in Adapt II (release 4) using i.v. citalopram from 379 subjects (unpublished data). Blood samples were collected at baseline and $6,15,21,30,36,45$, $60,90,150$, and $360 \mathrm{~min}$ after start of infusion, from an indwelling forearm catheter contralateral to the infusion catheter into vacutainers and centrifuged. Plasma was decanted, transferred to polypropylene tubes, and stored at $-80^{\circ} \mathrm{C}$. Racemic citalopram concentrations were determined using a high-performance liquid chromatographic technique developed in our laboratory (Foglia et al, 1997).

\section{Functional MRI}

During placebo and drug sessions, subjects completed three scans using a slightly modified version (consistent of angry, fearful, surprised and neutral $v s$ only angry and fearful emotional expressions) of our amygdala reactivity paradigm described in detail elsewhere (Brown et al, 2005). Briefly, the paradigm consisted of four blocks of a perceptual faceprocessing task interleaved with five blocks of a sensorimotor control task. During face processing, subjects viewed a trio of faces and matched one of two emotionally expressive (angry, fearful, or surprised) or neutral faces to an identical target. Stimuli across all three scans were all novel and derived from a standard set of emotional facial expressions (NimStim; www.macbrain.org). During the sensorimotor control, subjects viewed a trio of geometric shapes and selected one of two shapes identical to a target.

Blood oxygenation-level-dependent (BOLD) fMRI was acquired on a $1.5 \mathrm{~T}$ Signa Scanner (General Electric Medical
Systems, Milwaukee, WI) using an optimized reverse spiral sequence covering 28 axial slices $(3.8 \mathrm{~mm}$ thick) encompassing the entire cerebrum $(\mathrm{TR} / \mathrm{TE}=2000 / 35 \mathrm{~ms}, \mathrm{FOV}=$ $24 \mathrm{~cm}$, matrix $=64 \times 64)$. Data were processed in SPM2 using our standard protocol (Brown et al, 2005). Predetermined condition effects at each voxel were calculated using a $t$-statistic, producing a statistical image for face processing $v s$ sensorimotor control for each subject. Individual contrast images were used in second-level random-effects models accounting for scan-to-scan and subject-to-subject variability to determine task-specific regional responses at the group level.

The amygdala was chosen a priori as a region of interest (ROI) for all analyses, and defined using the Talairach Demon option of the WFU Pickatlas (Maldjian et al, 2003, 2004). Differences in activation were considered statistically significant at a voxel level of $p<0.01$ uncorrected and an extent of at least 10 contiguous voxels within our a priori ROI. Because of our a priori directionally specific hypotheses and our use of a rigorous random-effects model, these statistical thresholds effectively control for type I error arising from multiple comparisons (Forman et al, 1995).

Additionally, for each treatment, we examined the change in BOLD activation in the amygdala during the third scan (end of infusion) relative to the first scan (baseline) by including data from both these scans in a general linear model for each single subject. Resulting single-subject contrast images of the differential activation (ie 'scan $3>$ scan 1') of the amygdala during placebo and citalopram infusions were entered into a paired $t$-test wherein we examined the differential effect of treatment on changes in the magnitude and extent of BOLD activation between the baseline and end of infusion scans.

\section{Correlation Analyses}

Citalopram kinetics were modeled from plasma samples through a standard two-stage population approach with a three-compartment continuous infusion model using individual nonlinear regression in WinNonlin 4.0.1 (Pharsight Corporation, Mountain View, CA). Average single-subject concentrations associated with each scan were calculated based on the individual fitted parameters for the threecompartment citalopram pharmacokinetic model. These concentrations were then used in a linear regression with BOLD activation in amygdala voxels exhibiting a main effect of task using SPM ( $p<0.05$, FDR corrected across volume of amygdala, minimum 10 contiguous voxels). Because SPM2 uses a naive-pooled method (ie it treats each data point, or concentration and activation pair, as a separate individual), we confirmed the regression data using a linear mixedeffects model in NONMEM V (version 1.1, Fortran g77), which uses a Bayesian modeling approach that accounts for both inter-individual and intra-individual variability. The likelihood ratio test was used to discriminate between alternative models in NONMEM. The likelihood ratio test is based on the property that the ratio of the NONMEM objective function values ( $-2 \log$ likelihood) are asymptotically $\chi^{2}$ distributed. The objective function value is the sum of squared deviations between the predictions and the observations. An objective function decrease of 3.84 units was considered significant $\left(\chi^{2}\right.$, d.f. $\left.=1, p<0.05\right)$. Regression 
data were plotted using Prism (version 4.03; GraphPad Software Inc., San Diego, CA).

\section{RESULTS}

Subjects reported mild or no side effects, which did not differ between drug and placebo infusions. There were no remarkable differences in amygdala activation between scans during placebo infusion. There were only two small clusters in the left amygdala that decreased early in the placebo infusion $((-30,2,-24)$, cluster size $=13$ voxels, $T=3.90, p=0.004$, and $(-20,-6,-8)$, cluster size $=26$ voxels, $T=3.87, p=0.004)$. In contrast, mean amygdala
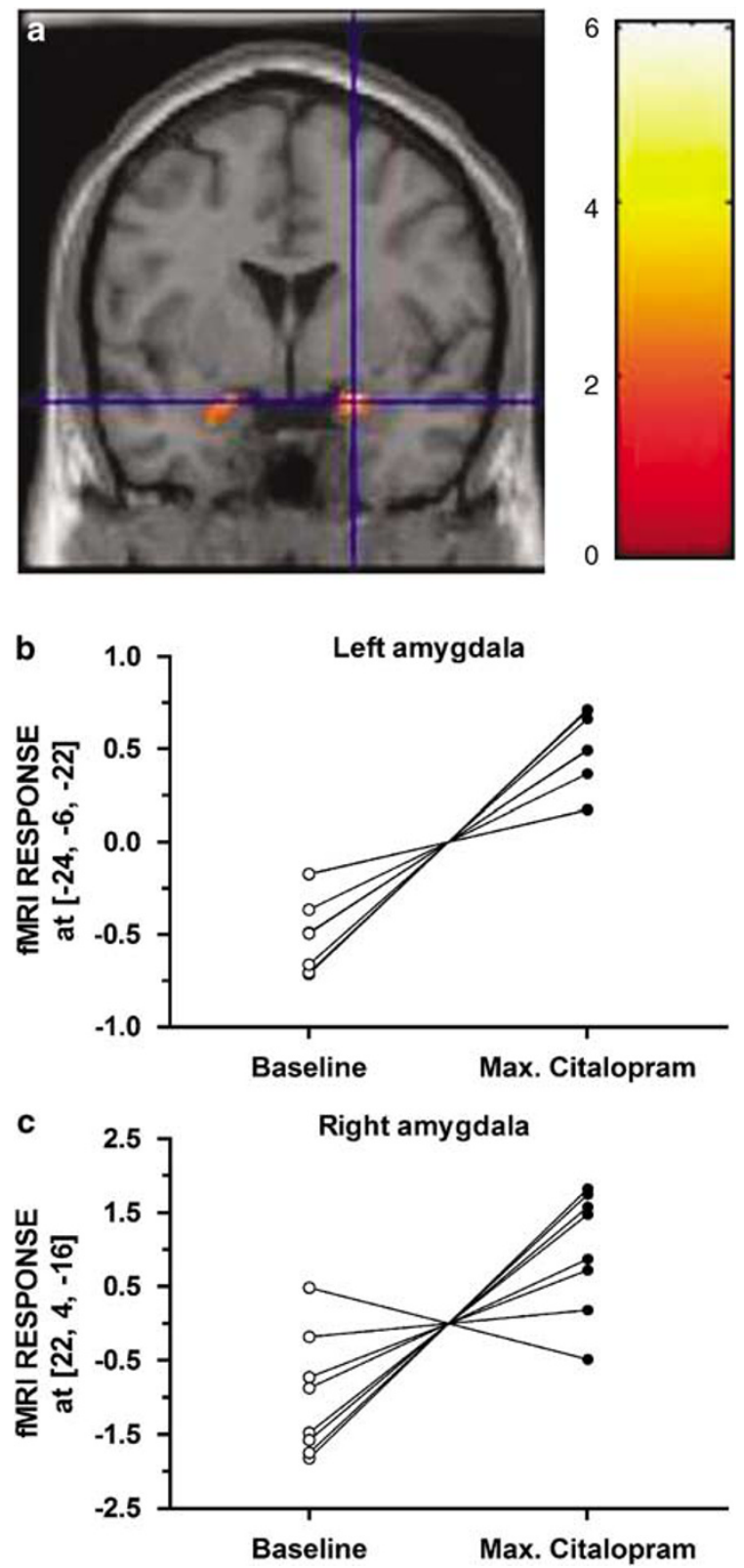

Figure I (a) Amygdala clusters exhibiting greater activation as a function of acute citalopram, Left amygdala $(-24,-6,-22)$ cluster size $=115$ voxels, $T=6.05, p<0.00 \mathrm{I}$; right amygdala $(22,4,-16)$ cluster size $=56$ voxels, $T=3.39, p=0.006$. Individual $\mathrm{fMRI}$ responses at baseline and at end of citalopram infusion for left amygdala (b) and right amygdala (c). reactivity significantly increased across successive scans during citalopram infusion. A cluster in the right amygdala exhibited increased activation early in the citalopram infusion compared to the baseline $((22,-4,-20)$, cluster size $=55$ voxels, $T=3.01, p=0.01)$. An even greater bilateral amygdala response during citalopram administration was found at the end of infusion, when drug concentrations approach their maxima, compared to baseline (Figure 1; left amygdala $(-24,-6,-22)$, cluster size $=$ 115 voxels, $T=6.05, p<0.001$; right amygdala $(22,4,-16)$ cluster size $=56$ voxels, $T=3.39, p=0.006$ ).

Direct comparisons in the differential activation of the amygdala between baseline and late infusion during placebo and citalopram administrations confirmed these observations. The increase in bilateral amygdala reactivity between late infusion and baseline is significantly greater during citalopram administration in comparison with placebo (left amygdala $(-26,-6,-26)$, cluster size $=52$ voxels, $T=5.75$, $p=0.001$ and right amygdala $(16,-6,-22)$, cluster size $=11$ voxels, $T=2.80, p=0.01)$. In fact, average amygdala activation significantly increased between baseline and late infusion during citalopram treatment (mean 1.081 , SD 0.658) whereas average amygdala activation decreased between baseline and late infusion during placebo (mean -0.5406, SD 1.108), (Figure 2).

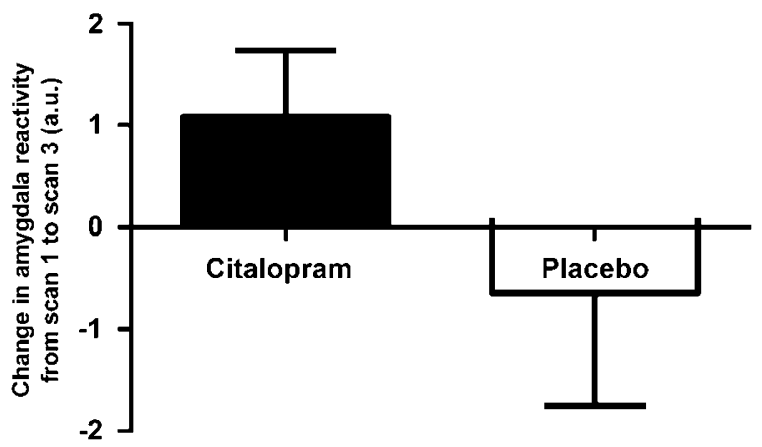

Figure 2 Change in left amygdala reactivity $(-26,-6,-26)$ between baseline and late infusion during citalopram and placebo. Paired $t$-test $(t=5.35, d f=7, p=0.00 \mathrm{I})$.

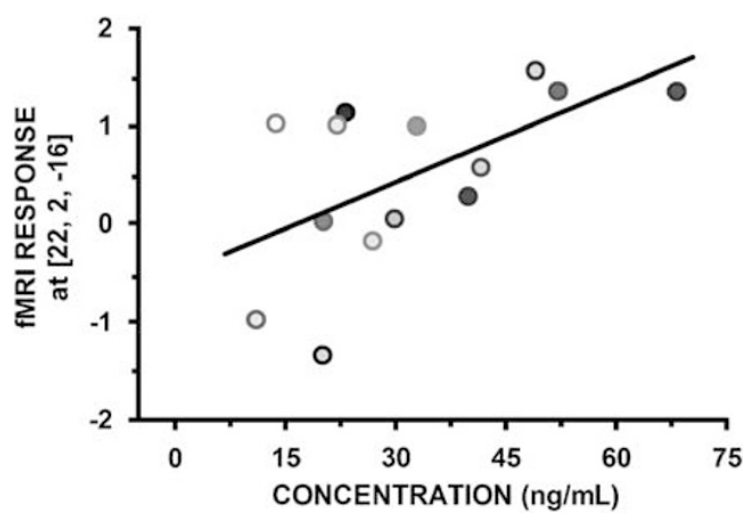

Figure 3 Amygdala reactivity correlates with citalopram concentration. Scans are color coded (time 2 is shaded, time 3 is solid). Citalopram concentrations could not be estimated for one subject due to missing plasma samples ( $n=7$ for this analysis only). 
Finally, linear regression analysis between scan-specific plasma citalopram concentrations and amygdala activation revealed a strong concentration-dependent increase in amygdala reactivity over infusion (Figure $3 ; r^{2}=0.334$, $p=0.0306)$. A separate linear mixed-effects model produced an identical $y$ intercept $(-0.51)$ and slope (0.03). The NONMEM objective function change between the initial model, which modeled BOLD as a constant with random effects (ie concentration was held constant), and the final model, which modeled both the slope (ie change in concentration) and intercept (ie baseline BOLD value) with random effects, was also significant (5.6 point decrease in objective function; $p=0.018$ ), which confirms the SPM linear regression (Figure 3).

\section{DISCUSSION}

Our data provide unique direct evidence that acute pharmacological 5-HT reuptake blockade potentiates the reactivity of the human amygdala to salient environmental stimuli. The data are consistent with both preclinical animal findings (Burghardt et al, 2004, 2007; Forster et al, 2006) and indirect indices of 5-HT effects on human amygdala function (Hariri et al, 2002; Heinz et al, 2005; Fisher et al, 2006; Rhodes et al, 2007). The current pattern of 5-HTmediated amygdala reactivity, which can drive behavioral and physiologic arousal, may represent an important pathway through which SSRIs achieve an antidepressant effect. Intriguingly, we hypothesize that our data may also reveal a mechanism contributing to clinical observations of extreme agitation, restlessness, and suicidal ideation in some individuals, especially children, during acute SSRI treatment (Bridge et al, 2007). This latter phenomenon may reflect rapid 5-HT-mediated amygdala sensitivity to environmental stimuli in the context of deficient mechanisms for behavioral control, which are largely supported by prefrontal circuitries exhibiting a more delayed response to increased 5-HT (Forster et al, 2006).

In fact, a recent study reported relative attenuation of amygdala reactivity after chronic oral administration of citalopram in healthy adults (Harmer et al, 2006). Such divergent acute and chronic effects of 5-HT reuptake blockade likely emerge in response to homeostatic shifts in serotonergic regulation of the functional balance between the amygdala and prefrontal cortex. While acute 5-HT reuptake blockade may potentiate stimulus-dependent amygdala reactivity, likely through stimulation of excitatory postsynaptic 5-HT receptors located on apical dendrites of glutamatergic neurons (McDonald and Mascagni, 2007), chronic blockade may lead to a more general increase in 5-HT neurotransmission via downregulation of negative feedback 5-HT autoreceptors (Blier and de Montigny, 1999). This shift toward generally increased neurotransmission after chronic reuptake blockade may allow 5-HT to potentiate the response of pyramidal neurons in prefrontal circuits, via volume transmission at excitatory 5-HT receptors localized extrasynaptically (Jansson et al, 2001; Sharp et al, 2007), thereby mediating top-down regulation of the amygdala (Hariri and Holmes, 2006).

While these results are exciting, with a remarkably consistent pattern of 5-HT reuptake blockade effects on amygdala activation across subjects, the small sample size greatly limits their inferential value and should be regarded as preliminary. Replication studies using this pharmacological fMRI design in larger samples with increased statistical power are needed to determine the robustness of our observed effects. Studies in clinical populations are also necessary to evaluate our speculations regarding the importance of this mechanism in the emergence of both therapeutic and aberrant behavioral responses during acute and chronic SSRI treatment. Through systematic extension of our preliminary results, a comprehensive model of how 5-HT modulates normal human amygdala function and its dynamic interactions with prefrontal regulatory circuits can be developed and used to inform our understanding of basic neurobehavioral processes. Additionally, such efforts may aid in understanding pathological amygdala function in psychiatric disorders, as well as predicting response to SSRIs and developing more effective 5-HT-based therapeutic interventions.

\section{ACKNOWLEDGEMENTS}

This research study was supported by NIH F31MH076420, K24MH065416, K23MH064678, K01MH072837, P41EB001975, and NARSAD. Support was also provided by the General Clinical Research Center of the University of Pittsburgh Medical Center (MO1RR000056) and the University of Pittsburgh Magnetic Resonance Research Center Pilot Imaging Program.

\section{DISCLOSURE/CONFLICT OF INTEREST}

In addition to the NIH grants listed above, Dr Pollock has received research support from Janssen Pharmaceuticals. He has served on the advisory board of Forest Laboratories and is a faculty member of the Lundbeck Institute. The other authors have no other disclosures or conflicts.

\section{REFERENCES}

Aizenstein HJ, Clark KA, Butters MA, Cochran J, Stenger VA, Meltzer CC et al (2004). The BOLD hemodynamic response in healthy aging. J Cogn Neurosc 16: 786-793.

Blier P, de Montigny C (1999). Serotonin and drug-induced therapeutic responses in major depression, obsessivecompulsive and panic disorders. Neuropsychopharmacology 21(2 Suppl): 91S-98S.

Bridge JA, Iyengar S, Salary CB, Barbe RP, Birmaher B, Pincus HA et al (2007). Clinical response and risk for reported suicidal ideation and suicide attempts in pediatric antidepressant treatment: a meta-analysis of randomized controlled trials. JAMA 297: 1683-1696.

Brown SM, Peet E, Manuck SB, Williamson DE, Dahl RE, Ferrell RE et al (2005). A regulatory variant of the human tryptophan hydroxylase- 2 gene biases amygdala reactivity. Mol Psychiatry 10: 805.

Burghardt NS, Bush DE, McEwen BS, LeDoux JE (2007). Acute selective serotonin reuptake inhibitors increase conditioned fear expression: blockade with a 5-HT(2C) receptor antagonist. Biol Psychiatry 62: 1111-1118.

Burghardt NS, Sullivan GM, McEwen BS, Gorman JM, LeDoux JE (2004). The selective serotonin reuptake inhibitor citalopram increases fear after acute treatment but reduces fear with chronic 
treatment: a comparison with tianeptine. Biol Psychiatry 55: $1171-1178$.

First MB, Spitzer RL, Gibbon M, Williams JBW (1995). Structured Clinical Interview for DSM-IV Axis I Disorders-Patient Edition (SCID-I/P, version 2.0). Biometrics Research Department, New York State Psychiatric Institute: New York.

Fisher PM, Meltzer CC, Ziolko SK, Price JC, Moses-Kolko EL, Berga SL et al (2006). Capacity for 5-HT1A-mediated autoregulation predicts amygdala reactivity. Nat Neurosci 9: 1362-1363.

Foglia JP, Pollock BG, Kirshner MA, Rosen J, Sweet R, Mulsant B (1997). Plasma levels of citalopram enantiomers and metabolites in elderly patients. Psychopharmacol Bull 33: 109-112.

Forman SD, Cohen JD, Fitzgerald M, Eddy WF, Mintun MA, Noll DC (1995). Improved assessment of significant activation in functional magnetic resonance imaging (fMRI): use of a cluster-size threshold. Magn Reson Med 33: 636-647.

Forster GL, Feng N, Watt MJ, Korzan WJ, Mouw NJ, Summers CH et al (2006). Corticotropin-releasing factor in the dorsal raphe elicits temporally distinct serotonergic responses in the limbic system in relation to fear behavior. Neuroscience 141: 1047-1055.

Hariri AR, Holmes A (2006). Genetics of emotional regulation: the role of the serotonin transporter in neural function. Trends Cogn Sci 10: 182-191.

Hariri AR, Mattay VS, Tessitore A, Kolachana B, Fera F, Goldman D et al (2002). Serotonin transporter genetic variation and the response of the human amygdala. Science 297: 400-403.

Harmer CJ, Mackay CE, Reid CB, Cowen PJ, Goodwin GM (2006). Antidepressant drug treatment modifies the neural processing of nonconscious threat cues. Biol Psychiatry 59: 816-820.
Heinz A, Braus DF, Smolka MN, Wrase J, Puls I, Hermann D et al (2005). Amygdala-prefrontal coupling depends on a genetic variation of the serotonin transporter. Nat Neurosci 8: 20-21.

Jansson A, Tinner B, Bancila M, Vergé D, Steinbusch HW, Agnati LF et al (2001). Relationships of 5-hydroxytryptamine immunoreactive terminal-like varicosities to 5-hydroxytryptamine-2A receptor-immunoreactive neuronal processes in the rat forebrain. J Chem Neuroanat 22: 185-203.

Maldjian JA, Laurienti PJ, Burdette JH (2004). Precentral gyrus discrepancy in electronic versions of the Talairach atlas. Neuroimage 21: 450-455.

Maldjian JA, Laurienti PJ, Kraft RA, Burdette JH (2003). An automated method for neuroanatomic and cytoarchitectonic atlas-based interrogation of fMRI data sets. Neuroimage 19: 1233-1239.

McDonald AJ, Mascagni F (2007). Neuronal localization of 5 -HT type $2 \mathrm{~A}$ receptor immunoreactivity in the rat basolateral amygdala. Neuroscience 146: 306-320.

Meyer-Lindenberg A, Buckholtz JW, Kolachana B, R Hariri A, Pezawas L, Blasi G et al (2006). Neural mechanisms of genetic risk for impulsivity and violence in humans. Proc Natl Acad Sci USA 103: 6269-6274.

Rhodes RA, Murthy NV, Dresner MA, Selvaraj S, Stavrakakis N, Babar S et al (2007). Human 5-HT transporter availability predicts amygdala reactivity in vivo. J Neurosci 27: 9233-9237.

Sharp T, Boothman L, Raley J, Quérée P (2007). Important messages in the 'post': recent discoveries in 5-HT neurone feedback control. Trends Pharmacol Sci 28: 629-636. 